\title{
The influence of naturalistic, directionally non- specific motion on the spatial deployment of visual attention in right-hemispheric stroke
}

\author{
Dario Cazzoli ${ }^{1,2, \#}$, Simone Hopfner ${ }^{3, \#}$, Basil Preisig ${ }^{3,4}$, Giuseppe Zito ${ }^{1}$, Tim \\ Vanbellingen $^{3,5}$, Michael Jäger ${ }^{1,6}$, Tobias Nef ${ }^{1,6}$, Urs Mosimann ${ }^{1,4}$, Stephan \\ Bohlhalter $^{3,5}$, René M. Müri ${ }^{1,3,7}$, and Thomas Nyffeler ${ }^{1,3,5^{*}}$
}

\section{Affiliations:}

${ }^{1}$ Gerontechnology \& Rehabilitation Group, University of Bern, Bern, Switzerland

${ }^{2}$ Nuffield Department of Clinical Neurosciences, University of Oxford, United Kingdom

${ }^{3}$ Perception and Eye Movement Laboratory, Departments of Neurology and Clinical Research, Inselspital, Bern University Hospital and University of Bern, Freiburgstrasse 10, 3010 Bern, Switzerland

${ }^{4}$ University Hospital of Old Age Psychiatry and Psychotherapy, University of Bern, Bern, Switzerland ${ }^{5}$ Neurology and Neurorehabilitation Center, Luzerner Kantonsspital, Luzern, Switzerland

${ }^{6}$ ARTORG Center for Biomedical Engineering Research, University of Bern, Bern, Switzerland

${ }^{7}$ Division of Cognitive and Restorative Neurology, Department of Neurology, Inselspital, Bern University Hospital and University of Bern, Switzerland

\# These authors contributed equally to this work.

* Corresponding author:

Prof. Dr. Thomas Nyffeler

Perception and Eye Movement Laboratory, Department of Neurology, Department of Clinical Research, Inselspital, Bern University Hospital and University of Bern, Freiburgstrasse 10, 3010 Bern, Switzerland

thomas.nyffeler@luks.ch

Fax: +41/(0)4120524 41

Tel.: +41/(0)41205 5686 


\section{Abstract}

An impairment of the spatial deployment of visual attention during exploration of static (i.e., motionless) stimuli is a common finding after an acute, right-hemispheric stroke. However, less is known about how these deficits: a) are modulated through naturalistic motion (i.e., without directional, specific spatial features); and, b) evolve in the subacute/chronic poststroke phase. In the present study, we investigated free visual exploration in three patient groups with subacute/chronic right-hemispheric stroke and in healthy subjects. The first group included patients with left visual neglect and a left visual field defect (VFD), the second patients with a left VFD but no neglect, and the third patients without neglect or VFD. Eye movements were measured in all participants while they freely explored a traffic scene without (static condition) and with (dynamic condition) naturalistic motion, i.e., cars moving from the right or left. In the static condition, all patient groups showed similar deployment of visual exploration (i.e., as measured by the cumulative fixation duration) as compared to healthy subjects, suggesting that recovery processes took place, with normal spatial allocation of attention. However, the more demanding dynamic condition with moving cars elicited different re-distribution patterns of visual attention, quite similar to those typically observed in acute stroke. Neglect patients with VFD showed a significant decrease of visual exploration in the contralesional space, whereas patients with VFD but no neglect showed a significant increase of visual exploration in the contralesional space. No differences, as compared to healthy subjects, were found in patients without neglect or VFD. These results suggest that naturalistic motion, without directional, specific spatial features, may critically influence the spatial distribution of visual attention in subacute/chronic stroke patients. 


\section{Keywords:}

Subacute/chronic Stroke; Hemispatial Neglect; Visual Attention Deployment; Motion; Eyemovements; Everyday relevant setting 


\section{Introduction}

Eye movements and visual attention move together across the visual space, at least in situations where the eyes are free to move (Hunt \& Kingstone, 2003). This phenomenon, often referred to as overt attention, is a fundamental behaviour during the activities of everyday life (Land, 2006). Cerebral lesions, in particular to the right hemisphere, can severely impair visual exploration and thus the spatial deployment of visual attention. Among the disorders following a right-hemispheric lesion, spatial neglect is a particularly disabling syndrome. Spatial neglect is commonly defined as a deficit in orienting towards, responding to, and reporting stimuli that are presented on the contralesional side of space (Heilman, Watson, \& Valenstein, 2003). In patients with acute stroke, this deficit in the spatial allocation of visual attention towards the contralesional space results in characteristic patterns during visual exploration. For instance, patients with neglect present a significant ipsilesional bias in the spatial distribution of visual fixations, a bias of early attentional orientation towards the ipsilesional space, frequent re-fixations (i.e., repeated fixations over the same region), and impaired saccade metrics (Karnath, Niemeier, \& Dichgans, 1998; Mort \& Kennard, 2003; Müri, Cazzoli, Nyffeler, \& Pflugshaupt, 2009; Niemeier \& Karnath, 2000; Sprenger, Kompf, \& Heide, 2002). In approximately $75 \%$ of neglect patients, patterns of visual attention allocation in space normalise within six months after stroke (Stone, Patel, Greenwood, \& Halligan, 1992). In these patients, visual exploration is characterized by a re-orientation of visual attention towards the contralesional side, while only an early attentional bias (i.e., first saccade) towards the ipsilesional side may persist (Karnath, 1988; Mattingley, Bradshaw, Bradshaw, \& Nettleton, 1994; Pflugshaupt et al., 2004; Pizzamiglio et al., 1992).

Several factors can influence the allocation of attention in space, and these can have a spatial or non-spatial nature. Motion, which is an omnipresent feature of visual stimuli in everyday situations, is one of those factors (Zihl, 1995). For instance, visual attention can improve 
when patients with neglect are instructed to look at dots on a dark background, all moving coherently toward the contralesional space (Kerkhoff et al., 2014). Furthermore, in acute neglect patients, it could be shown that moving stimuli in the contralesional space can be a spatial cue and attract visual attention (Butter, Kirsch, \& Reeves, 1990; Mattingley, Bradshaw, \& Bradshaw, 1994; Plummer, Dunai, \& Morris, 2006). Nevertheless, the direction of the modulatory effect of motion seems also to depend on the integrity of the optic radiation, i.e., the presence or absence of an additional visual field defect (VFD). In a recent study, we showed that neglect patients with an additional VFD may present increased or unchanged neglect severity when confronted with motion in a touchscreen-based cancellation task (Hopfner et al., 2015).

On the other hand, patients with VFD but no neglect may show a reversed spatial bias during visual exploration, i.e., they may produce an increased number of gaze shifts towards the affected side of space (Ishiai, Furukawa, \& Tsukagoshi, 1987; Pambakian et al., 2000). This contralesional spatial bias is thought to reflect an attempt to compensate for VFD, and seems also to increase with higher task demands (Hardiess, Papageorgiou, Schiefer, \& Mallot, 2010).

However, to date far less is known about the effects on the spatial attentional allocation in right-hemispheric patients of motion that has no specific, directional spatial features, i.e., of naturalistic motion in everyday scenes, where elements move in both the ipsi- and the contralesional space, in heterogeneous directions.

In the present study, we thus adopted a virtual reality (VR) approach, and established an innovative setup with large projection displays. We examined free visual exploration of a virtual traffic scene, without (static condition) and with (dynamic condition) naturalistic motion, in which cars could move from the left to the right or vice versa. This not only allowed the stimulation of the full field of view (i.e., $180^{\circ}$; as opposed to a small stimulus array, limited by the computer screen), but also the assessment of visual exploration in the 
extrapersonal, far space (as opposed to the peripersonal, near space, which is commonly assessed in paper-pencil and computer-based tests). Both aspects contribute to a more naturalistic and ecologically valid evaluation of the effects of motion. Furthermore, there is little knowledge about the modulatory effects of motion in patients with a subacute/chronic stroke, and about the specificity of the interactions with VFD. For this reason, in the present study, we included patients with subacute/chronic left-sided neglect and VFD and, as control groups, patients with a subacute/chronic left VFD but no neglect, and patients with righthemispheric lesions but no neglect or VFD. Additionally, healthy subjects also performed the task.

Based on previous research, we hypothesized that the influence of naturalistic, directionally non-specific motion on the spatial allocation of visual attention would differ between patient groups and healthy subjects. In particular, we expected that, in subacute/chronic neglect patients with VFD, neglect severity would increase in the dynamic condition. 


\section{Methods}

\subsection{Subjects}

Twenty-four patients who suffered from a first ischemic or haemorrhagic right hemispheric stroke (aged between 25 and 76, mean $=54.20, \mathrm{SD}=13.27 ; 8$ women) and 8 healthy subjects (aged between 25 and 78, mean $=60.67, \mathrm{SD}=19.43 ; 3$ women) were included in the study after giving written, informed consent. There was no statistically significant difference between patients and healthy subjects with respect to age $(t(30)=-1.019, p=.316 ; 2$-tailed $)$ or gender $\left(\chi^{2}(1)=.046, p=.575\right)$. The group of twenty-four patients was comprised of 8 patients with left-sided visual neglect and VFD, 8 patients with a left-sided VFD but without neglect, and 8 patients without any neglect or VFD. The mean interval between stroke onset and testing was 589 days (range: $28-1737$ days, $\mathrm{SD}=613$ ). No statistically significant differences were found between the three patient groups with respect to the mean interval between stroke onset and testing $(F(2,21)=.932, p=.409)$, age $(F(2,21)=.231, p=.796)$, or gender $\left(\chi^{2}(2)=.375 ; p=.829\right)$. The definition of the beginning of the chronic post-stroke period is variable among sources in the literature. Although some sources define the beginning of the chronic stroke period relatively late (i.e., 180 days after stroke), other sources set the beginning of this period already at three weeks after stroke (e.g., Allen, Hasso, Handwerker, \& Farid, 2012). All patients were included in the present study at the earliest 28 days after stroke, thus overlapping with either the subacute or the chronic post-stroke phase, depending on the applied definition. In order to reflect the non-absolute nature of this definition, we opted to name the post-stroke phase with the more general term "subacute/chronic".

Since patients were in the subacute/chronic stage after stroke, neglect diagnosis was based not only on standard neuropsychological testing, but primarily on the observation of neglect manifestations in everyday behaviour. Other studies based neglect diagnosis primarily on the 
presence of neglect signs in computerized and/or paper-pencil tests (e.g., in at least one or two of these tests). While the present study aimed at a more naturalistic and ecologically valid evaluation of the effects of motion in neglect (and thus stressed the importance of neglect manifestations in everyday behaviour), it is to note that the definition of different criteria may lead to the inclusion/exclusion of different patients, and thus to potential differences in the results. For the assessment of neglect manifestations in everyday behaviour, the Catherine Bergego Scale was applied, which is able to quantify the influence of neglect-related persisting deficits in the activities of daily living (Azouvi, 1996; Azouvi et al., 2003). Two classes of neuropsychological tests were also administered: a cancellation task (either Bells test [Gauthier, Dehaut, \& Joanette, 1989] or Star Cancellation test [Wilson, Cockburn, \& Halligan, 1987]), and a line bisection task (Wilson et al., 1987). For the Bells test, we calculated the number of targets omitted on the left side minus the number of targets omitted on the right side, and the cut-off score was set at $>2$ (according to Azouvi et al., 2006). For the Star cancellation test, the cut-off score was set at $15 \%$ of omitted left-sided targets (according to Ferber \& Karnath, 2001), i.e., 4 left-sided target omissions. For the line bisection task, a mean rightward deviation equal to or larger than $11 \%$ from the actual midline was considered as clinically relevant (according to Schenkenberg, Bradford, \& Ajax, 1980). Since an evaluation of whether neglect patients also have an additional VFD is difficult to achieve with clinical confrontation testing or visual perimetry (Kerkhoff \& Schindler, 1997; Müller-Oehring et al., 2003), we also assessed whether the right optic radiation was damaged or not by means of a track-wise 'hodological' lesion-deficit analysis (Thiebaut de Schotten et al., 2014), which is based on a recently published DTI atlas (Thiebaut de Schotten et al., 2011; Rojkova et al., 2016). The atlas provides the probability for each voxel in the MNI space to belong to a specific white matter tract. To conduct this analysis, we used the 'Tractotron' software (Thiebaut de Schotten et al., 2014). In a first step, we mapped the individual lesions of the patients in the structural MRI data by means of the MRIcron software (Rorden, 
Karnath, \& Bonilha, 2007). The same procedure as in Karnath, Fruhmann Berger, Küker and Rorden (2004) and Karnath, Himmelbach and Rorden (2002) was applied: if an MRI was conducted within the $48 \mathrm{~h}$ post-stroke, then diffusion-weighted scans were used, otherwise T2-weighted scans were used. We delineated the boundary of the lesions directly on the individual MRI images, for each transverse slice. We then mapped the scans and the lesions into approximate Montreal Neurological Institure (MNI) space, applying the spatial normalisation algorithm provided by SPM5 (http://www.fil.ion.ucl.ac.uk/spm/). Only CT scans were available for some patients. In this case, we performed lesion mapping directly on the T1-weighted MNI single-subject template with a resolution of 1x1x1 mm, as implemented in MRIcron (Rorden \& Brett, 2000). Lesion mapping was performed by a collaborator who was naïve with respect to patients' behavioural performance, and the accuracy was then checked by a second, independent collaborator. In a second step, we then performed the trackwise 'hodological' lesion-deficit analysis with the 'Tractotron' software (Thiebaut de Schotten et al., 2014). The patients' individual lesion maps were overlapped with the map of the right optic radiation in order to analyse the individual pattern of integrity of the latter. The optic radiation was considered to be disconnected based on a binary measure, i.e., considering the optic radiation to be damaged if the patients' individual lesion map overlapped with a voxel within the optic radiation map with a probability greater than $50 \%$ (i.e., above chance level). The analysis revealed that all patients in the group with neglect and VFD, and all patients in the group with VFD only (i.e., no neglect) had a damage to the right optic radiation. Conversely, none of the patients in the group without any VFD or neglect had damage to the right optic radiation. As an illustration, Figure 1 depicts the localisation of the brain lesions and their degree of overlap, transferred to standard atlases and with respect to the right optic radiation, in the three groups of patients. 
Figure 1

about here

In patients with VFD and without neglect, the visual field was assessed by means of perimetry (Octopus Perimetry or Goldman Kinetic Perimetry; Octopus Perimeter 101, Haag-Streit International, Bern-Köniz, Switzerland).

All participants (i.e., patients and healthy subjects) had normal or corrected-to-normal visual acuity. Demographic and clinical details of patients are presented in Table 1.

Table 1

about here

Ethical approval to conduct the present study was provided by the Ethics Committee of the States of Bern and Lucerne. The study was carried out in accordance with the principles laid down in the latest version of the Declaration of Helsinki.

\subsection{Stimulus material and apparatus}

The stimulus material consisted of a virtual traffic scene, displaying an intersection environment, with a two-way road and a pedestrian-crossing, surrounded by buildings and trees. The technical details and a usability evaluation are published elsewhere (Jäger, Nyffeler, Müri, Mosimann, \& Nef, 2015). The virtual scene was presented under two 
conditions: a static and a dynamic condition. In the static condition, the virtual scene was presented without any road traffic, i.e., only the motionless environment, without any cars, was displayed. In the dynamic condition, the same virtual scene was presented as background. However, the dynamic condition also included cars, moving from the left to the right and from the right to the left on the main road. The number of cars driving from the left to the right and vice versa was counterbalanced within each trial, with all cars moving at the same and constant speed. To reduce the potential influence of specific features of the cars (i.e., shape, colour), only cars of the same model and colour were selected (specifically, Ford Mondeo, white). Figure 2 shows an example of the virtual traffic scene in the dynamic condition.

Figure 2

about here

The virtual scenes were projected by three overhead projectors (Ultra Short Focus LCD projector, Sanyo, Moriguchi Osaka, Japan), with a resolution of $1024 x 768$ pixels, onto three canvas projection screens $(1.80 \times 1.39 \mathrm{~m}$ each), installed in front of the participant (Fig. 3). Screen 2 and 3 were tilted by $120^{\circ}$ with respect to Screen 1, and thus creating a field of view of $180^{\circ}$ (horizontal) by $40^{\circ}$ (vertical). Participants were placed in front of Screen 1 , with a viewing distance of $155 \mathrm{~cm}$. The simulation was controlled by three computers running Microsoft Windows 7 operating system (Microsoft Corp., Redmond, WA). One of the computers calculated and controlled the dynamic aspects of the virtual scene in the dynamic condition, whereas the other two computers refreshed the image at a rate of $35 \mathrm{~Hz}$. 
Figure 3

about here

\subsection{Eye-movement recording}

Eye movements were recorded using an infrared, head-mounted eye-tracking system (SMI iView X HED, SensoMotoric Instruments GmbH, Teltow, Germany), with a sampling rate of $50 \mathrm{~Hz}$, a spatial resolution of typically $<0.1^{\circ}$, and a tracking accuracy of typically $0.5^{\circ}-1^{\circ}$ (largely depending on calibration accuracy). The eye-tracking system is composed by two cameras, which are mounted on a helmet. The first camera records the scene in front of the person wearing the helmet, with a field of view of $50^{\circ}$ (horizontal) by $40^{\circ}$ (vertical), corresponding to his/her line of sight. The second camera measures monocularly the position of the pupil and of the corneal reflection, thus allowing to compute the direction of gaze. In the present study, gaze direction was recorded from the right eye, and the system allowed participants to freely move their head. In order to analyse which part of the virtual traffic scene was fixated in each recorded time frame, the information coming from the two cameras was merged, i.e., gaze direction recorded by one camera was superimposed to the visual scene recorded by the other camera. The resulting data sets thus consisted of individual video sequences and a data file, including - for each time frame - a cursor indicating the current point of gaze on the currently explored part of the visual scene. For data pre-processing, the recorded video sequence and the corresponding data file were uploaded in $\mathrm{BeGaze}^{\mathrm{TM}}$ analysis software (SensoMotoric Instruments GmbH, Teltow, Germany). Fixation detection threshold was set at a minimal duration of $100 \mathrm{~ms}$ and a maximal dispersion of 100 pixels. Individual gaze position was mapped into the reference space by means of the SMI Semantic Gaze 
Mapping analysis software tool (SensoMotoric Instruments GmbH, Teltow, Germany). This software enables to map the location of individual gaze positions in the video recordings made by single participants, fixation-by-fixation, on a common reference image. The resulting output is represented by a data file in which each fixation of each participant is associated with an exact location, defined on the $\mathrm{x}$ - and y-coordinates, in pixels, on a reference image. In the present study, the reference image was a png file, depicting the whole intersection environment (1500 x 368 pixels) used as the virtual scene during the experiment.

\subsection{Experimental procedure}

Participants were seated in front of the three projection screens, with their mid-sagittal plane aligned with the centre of the middle projection screen (screen 1; see Fig. 3). To ensure the accuracy of gaze position tracking, a 5-point calibration procedure was carried out, in which participants were asked to fixate 5 dots presented at different locations on the central projection screen, in a fixed order. During the calibration process, participants were instructed to follow the dots with their eyes only, and to hold their head in a steady position. Once the calibration procedure was completed, participants were allowed and encouraged to freely move their head.

Participants were then asked to freely explore the traffic environment under the two experimental conditions (i.e., the static and the dynamic condition). To familiarize the participants with the experimental setting, a practice sequence from the static and the dynamic condition was presented prior to the experiment proper.

The static and the dynamic conditions were administered in the same order for each participant, i.e., the static condition followed by the dynamic condition. The static condition entailed the presentation of the static, virtual traffic scene during 2 minutes. The dynamic condition was comprised of 15 sequences, where the speed and the direction of the car were 
presented in random order, lasting approximately 16 seconds each, and separated by a black screen during 4 seconds. Participants were allowed a break of approximately 10 minutes between the static and the dynamic condition. The overall duration of the experiment was of approximately 16 minutes.

\subsection{Data analysis}

In a first analysis, we aimed to assess early attentional orienting during free visual exploration. For this purpose, we calculated the mean gaze position during early attentional orienting, i.e., the mean coordinate, on the x-axis, of the visual fixation(s) occurring during the first second of visual exploration in the static and the dynamic condition, respectively. The data concerning the mean gaze position during early attentional orienting underwent a mixedmodel analysis of variance (ANOVA), with the within-subjects factor 'condition' (levels: static; dynamic) and the between-subjects factor 'group' (levels: patients with neglect and VFD; patients with VFD only; patients with right-hemispheric lesions without neglect or VFD; healthy subjects). Moreover, for every participant, we also evaluated whether the mean gaze position during early attentional orienting was located in the left or the right hemispace. For the static and the dynamic condition, we tested whether there was a significant association between the group (patients with neglect and VFD; patients with VFD only; patients with right-hemispheric lesions without neglect or VFD; healthy subjects) and the location of the mean gaze position during early attentional orienting (left hemispace; right hemispace) by means of a Pearson's chi-square test (with Fisher's exact test method).

In a second analysis, we assessed the spatial distribution of visual exploration throughout the task, in both the static and the dynamic conditions. For this purpose, the cumulative fixation duration (CFD), i.e., the sum of the duration of all visual fixations, was computed over six vertical columns, covering the whole field of view: leftmost (from $-90^{\circ}$ to $-60^{\circ}$ ), left (from - 
$60^{\circ}$ to $-30^{\circ}$ ), left central (from $-30^{\circ}$ to $0^{\circ}$ ), right central (from $0^{\circ}$ to $30^{\circ}$ ), right (from $30^{\circ}$ to $60^{\circ}$ ), rightmost (from $60^{\circ}$ to $90^{\circ}$ ). The distribution of the CFD over these six columns was calculated with Matlab (7.8.0.347, Mathworks Inc., Natick MA), converting the degrees of visual angle to portions of the reference image (1500 pixels on the horizontal dimension in total): leftmost ( 0 - 249 pixels), left ( $250-499$ pixels $)$, left central (500 - 749 pixels $)$, right central (750 - 999 pixels), right (1000 - 1249 pixels), rightmost (1250 - 1500 pixels). Since the presentation times in the static and the dynamic conditions were not of equal length, a direct comparison of the absolute distribution of the CFD over the six columns was not practicable. We thus decided to convert the absolute CFD values over the six columns into percentage CFD values, in both the static and the dynamic condition, according to the formula: [(absolute CFD in a given column / sum of the absolute CFD over columns 1 to 6)*100]. In a first step, the percentage CFD data underwent an omnibus, mixed-model ANOVA, with the within-subjects factors 'condition' (levels: static; dynamic) and 'column' (levels: leftmost; left; left central; right central; right; rightmost), and the between-subjects factor 'group' (levels: patients with neglect and VFD; patients with VFD only; patients with right-hemispheric lesions without neglect or VFD; healthy subjects). In a second step, in order to investigate the source of significant interactions, we performed post-hoc ANOVAs, adjusting the significance levels according to the Bonferroni procedure (i.e., dividing $\alpha$ by the number of performed post-hoc ANOVAs).

All subsequent post-hoc tests were conducted by means of Bonferroni-corrected $t$-tests. 


\section{Results}

\section{Early attentional orienting}

The analysis of the mean gaze position during early attentional orienting revealed a significant main effect of the factor 'group' $(F(3,28)=4.019, p=.017)$, but no significant effect of the factor 'condition' or of the interaction 'condition x group' (all $p$ 's $>.737$ ). Irrespective of the dynamic or static condition, and as indicated by Bonferroni-corrected post-hoc tests, the mean gaze position during early attentional orienting was significantly rightward-deviated in patients with neglect and VFD in comparison to all other groups (i.e., patients with VFD only, patients with right-hemispheric lesions without neglect or VFD, and healthy subjects). The results concerning the mean gaze position during early attentional orienting and the corresponding post-hoc tests are depicted in Figure 4.

\section{Figure 4}

about here

Moreover, for every participant, we also evaluated whether the mean gaze position during early attentional orienting was located in the left or the right hemispace. For the static condition, the mean gaze position during early attentional orienting was located in the right hemispace in all patients with neglect and VFD, whereas this was the case for 3 patients with VFD only (left hemispace for 5 patients), for 3 patients with right-hemispheric lesions without neglect or VFD (left hemispace for 5 patients), and for 2 healthy subjects (left hemispace for 6 healthy subjects). There was a significant association between the group and whether the mean gaze position during early attentional orienting was located in the left or the right 
hemispace $\left(\chi^{2}(3)=11.456, p=.012\right.$, Fisher's exact test). Direct pairwise testing evidenced that this association was significant when considering the group of patients with neglect and VFD in comparison to all other groups (all $p$ 's $<.026$ ). The odds of the mean gaze position during early attentional orienting being located in the right hemispace was thus higher in the group of patients with neglect and VFD than in the groups of patients with VFD only, of patients with right-hemispheric lesions without neglect or VFD, or of healthy subjects. For the dynamic condition, the mean gaze position during early attentional orienting was located in the right hemispace in 7 patients with neglect and VFD (left hemispace for 1 patient), whereas this was the case for 2 patients with VFD only (left hemispace for 6 patients), for 2 patients with right-hemispheric lesions without neglect or VFD (left hemispace for 6 patients), and for 2 healthy subjects (left hemispace for 6 healthy subjects). There was a significant association between the group and whether the mean gaze position during early attentional orienting was located in the left or the right hemispace $\left(\chi^{2}(3)=9.080, p=.033\right.$, Fisher's exact test). Direct pairwise testing evidenced that this association was significant when considering the group of patients with neglect and VFD in comparison to all other groups (all $p$ 's $<.041$ ). The odds of the mean gaze position during early attentional orienting being located in the right hemispace was thus higher in the group of patients with neglect and VFD than in the groups of patients with VFD only, of patients with right-hemispheric lesions without neglect or VFD, or of healthy subjects.

\section{Cumulative fixation duration (CFD) spatial distribution}

The omnibus ANOVA on the percentage CFD data revealed a significant main effect of factor 'column' $(F(5,140)=26.731, p<.001)$, significant two-way interactions between factors 'column x group' $(F(15,140)=4.393, p<.001)$ and 'condition $\mathrm{x}$ column' $(F(5,140)=$ $41.179, p<.001$ ), and a significant three-way interaction between factors 'condition $\mathrm{x}$ column x group' $(F(15,140)=2.364, p=.005)$. No other main effects or interactions were 
significant (all $p$ 's $>$.999). In order to investigate the source of the significant three-way interaction in the omnibus ANOVA, we performed two separate post-hoc ANOVAs for the static and the dynamic conditions (significance level set at $p=.05 / 2=.025$ ). In the static condition, there was a significant main effect of the factor 'column' $(F(5,140)=47.564, p<$ .001 ), but no significant effect of the factor 'group' or of the two-way interaction 'column $\mathrm{x}$ group' (all p's $>.151$ ). In contrast, in the dynamic condition, there was a significant main effect of factor 'column' $(F(5,140)=20.012, p<.001)$ and of the two-way interaction 'column x group' $(F(15,140)=5.382, p<.001)$, but no significant effect of the factor 'group' $(p>$.999). This indicates that the distribution of the percentage CFD over columns was different among groups in the dynamic condition, but not in the static one. In order to investigate the source of the significant two-way interaction in the dynamic condition, we performed separate post-hoc one-way ANOVAs for each of the six columns (significance level set at $p=.05 / 6=.008$ ). This analysis revealed a significant effect of the factor 'group' for the leftmost column $(F(3,28)=11.859, p<.001)$, but not for any other column (all $p$ 's $>$ .009). As indicated by Bonferroni-corrected post-hoc tests, in the leftmost column, patients with neglect and VFD showed significantly lower percentage CFD values than healthy subjects, whereas patients with VFD only showed significantly higher percentage CFD values than all other groups (i.e., patients with neglect and VFD, patients with right-hemispheric lesions without neglect or VFD, and healthy subjects). The results concerning the percentage CFD and the corresponding post-hoc tests are depicted in Figure 5.

Figure 5

about here 


\section{Discussion}

The present study analysed free visual exploration behaviour in a static and dynamic, naturalistic virtual traffic scene in three groups of subacute/chronic right-hemispheric stroke patients and in healthy subjects. The first group included patients with left visual neglect and a probable VFD due to an additional lesion to the optic radiation, the second included patients with a left VFD only, and the third included patients without neglect or VFD.

In the static condition, the spatial distribution of CFD was similar across all patient groups and the group of healthy subjects. In the dynamic condition, however, neglect patients with an additional VFD showed a significant decrease of CFD in the contralesional, peripheral space, whereas patients with only VFD showed a significant increase of CFD in the contralesional, peripheral space. No differences compared to healthy subjects were found in patients without neglect or VFD.

Previous studies of visual exploration with free head and eye movements found stable patterns in healthy individuals, evidencing a symmetrical and bell-shaped distribution of visual fixations in space (Karnath \& Fetter, 1995; Karnath, Fetter, \& Dichgans, 1996; Karnath et al., 1998). In the present study, this bell-shaped fixation distribution was also observed in the static condition: longer CFD were found on the central columns (i.e., left central and right central), and shorter CFD on more peripheral columns (i.e., leftmost, left, right, rightmost). Remarkably, all three patient groups showed a similar fixation distribution compared to healthy subjects in the static condition. This finding probably reflects recovery processes, which have been shown to take place in the subacute/chronic stroke phase (Nyffeler et al., 2011; Nyffeler, Müri, Pflugshaupt, Wartburg, \& Hess, 2006; Werhahn, Conforto, Kadom, Hallett, \& Cohen, 2003), restoring a normal spatial deployment of attention, at least in less demanding, static situations. However, the analysis of early attentional orienting evidenced that the mean gaze position was significantly rightward-deviated in patients with neglect and 
VFD in comparison to all other groups, irrespective of the condition, i.e., during both static and dynamic visual scenes exploration. This finding is in line with the results of previous studies, showing that the direction of early attentional orienting during free visual exploration or search is sensitive to the presence of neglect, even if residual or partially recovered (in terms of more ipsilesional than contralesional first saccades; e.g., Olk, Harvey, \& Gilchrist, 2002; Pflugshaupt et al., 2004; Gainotti, De Luca, Figliozzi, \& Doricchi, 2009; Müri, Cazzoli, Nyffeler, \& Pflugshaupt, 2009; Cazzoli, Nyffeler, Hess, \& Muri, 2011; but also see Niemeier \& Karnath, 2000; Ro, Rorden, Driver, \& Rafal, 2001).

As a new finding, however, non-homogenous motion, with no specific, directional spatial features, such as in naturalistic scenes, triggered different patterns in the distribution of CFD in the four groups. Healthy subjects were able to flexibly shift their spatial attention allocation towards relevant areas of the stimulus display, in order to efficiently gather information. They showed a redistribution of the CFD from the central columns, as observed in the static condition, towards the more peripheral columns. Such a redistribution is congruent and appropriate with respect to task demands, since, in the dynamic condition, central areas did not convey important information about the traffic situation (i.e., cars were driving from the periphery towards the centre).

In neglect patients with an additional VFD, the dynamic condition triggered a rightward exploration bias, with a significantly shorter CFD in the leftmost column as compared to healthy subjects. Hence, the typical deficit in directing visual attention towards the contralesional side of space, with a resulting ipsilesional bias (Behrmann, Watt, Black, \& Barton, 1997; Chedru, Leblanc, \& Lhermitte, 1973; Karnath, 1994; Müri et al., 2009), which was absent in the static condition, re-emerged in the dynamic condition. This suggests that the dynamic condition with naturalistic, directionally non-specific motion imposed higher demands on the mechanisms directing attention in space, which could not be compensated and thus led to the re-appearance of spatial attentional deficits. The new finding that 
subacute/chronic neglect patients with an additional VFD demonstrated a rightward shift in their attentional allocation in the far space when naturalistic, directionally non-specific moving stimuli were presented is interesting in the context of results obtained in patients with acute stroke in the near space (Hopfner et al., 2015). Using another approach, with a simple touchscreen-based cancellation task, the behaviour of neglect patients was analysed under static and different dynamic conditions. In neglect patients with an intact optic radiation, the severity of neglect significantly decreased when dynamic stimuli were presented, whereas it significantly increased in neglect patients with an additional damage to the optic radiation. In line with this finding, in the present study all neglect patients presented an additional damage to the optic radiation, as evidenced by a probabilistic track-wise lesion analysis. To explain this phenomenon, we postulate (see also Hopfner et al., 2015) that, in neglect patients, an additional, left-sided visual field defect may prevent the cueing effect exerted by left-sided moving stimuli, thus allowing right-sided moving stimuli to more readily capture attention, and thus increasing neglect severity.

In the control group of patients with VFD only (i.e., no neglect), on the other hand, an opposite visual exploration pattern was found in the dynamic condition. Patients showed a significant increase of the CFD in the leftmost column, as compared to all other groups. Previous research, investigating visual exploration with different tasks, has consistently evidenced abnormal scanning behaviour in patients with VFD (Hardiess et al., 2010; Mort \& Kennard, 2003; Pambakian et al., 2000; Tant, Cornelissen, Kooijman, \& Brouwer, 2002; Zihl, 1995). In VFD patients, visual exploration is often biased towards the affected side of space, indicating compensatory strategies (Ishiai et al., 1987; Pambakian et al., 2000). Importantly, this compensatory shift of visual exploration depends on task demands (Hardiess et al., 2010). The higher the task demands, the more the patients bias their visual exploration towards the affected side of space. In line with this view, we thus suggest that, in the present study, the more demanding dynamic condition with naturalistic, directionally non-specific motion 
triggered a more active compensatory strategy than the static condition in VFD patients, in order to align a larger amount of critical visual information with the intact hemifield. The results of a recent driving simulation study, in which the visual exploration behaviour of patients with VFD was analysed, are also in line with this interpretation (Papageorgiou et al., 2012). In this study, patients were asked to adapt their driving speed in order to safely cross an intersection in a virtual car. Successful driving behaviour in more demanding traffic situations was characterized by an increased amount of exploratory eye movements towards the blind hemifield.

The visual exploration behaviour observed in patients without neglect or VFD was similar to the one of healthy subjects, as the fixation distribution in the peripheral columns was more balanced than in neglect patients with an additional VFD and in patients with VFD only.

Although free visual exploration presents high ecological validity in assessing the spatial deployment of visual attention, some elements of caution should also be considered in the interpretation of the results. First, some previous studies (e.g., Karnath \& Fetter, 1995; Karnath et al., 1996; Karnath et al., 1998) recorded eye movements in neglect patients during the visual search of a non-existing target, thereby giving the participants an incentive to actively explore the entire scene (i.e., to find the target). In the current study, in contrast, participants were merely asked to freely explore the traffic scene. Thus, it is possible that the differences between conditions in the spatial distributions of fixations are not only attributable to the presence or absence of motion per se, but also to strategy differences, i.e., participants would mostly focus on the central part of the scene in the static condition, and actively explore the entire scene only when triggered to do so by motion cues. This would also be in line with the behaviour observed in neglect patients, as their spatial deficit is known to depend on the size of the actively explored area (e.g., Karnath \& Niemeier, 2002). Second, some neglect patients seem to be able to direct their gaze towards left-sided targets in search tasks, but may fail to acknowledge the presence of these targets (e.g., by a button press; see, e.g., 
Benson, Ietswaart, \& Milner, 2012; Ladavas, Zeloni, Zaccara, \& Gangemi, 1997; Bourgeois et al., 2015), suggesting that attention and gaze shifts may not always be strictly coupled in neglect.

To sum up, the present study shows that the spatial deployment of visual attention is significantly influenced by motion in a setting simulating an everyday relevant situation. Moreover, the results suggest that, in subacute/chronic stroke patients with 'remitted' symptoms, biases in attentional allocation might re-emerge when attentional demands increase with naturalistic, directionally non-specific moving stimuli. Under higher attentional demands, an ipsilesional bias may re-emerge in neglect patients with additional VFD, whereas a contralesional bias may emerge in patients with VFD only. Thus, motion seems to influence the allocation of visual attention differentially.

The present findings may also have practical implications. For instance, it is conceivable that neglect patients with VFD in a subacute/chronic post-stroke stage may be able to compensate for their deficits in static situations, but may present again neglect symptoms when confronted with naturalistic, moving stimuli. This is particularly relevant for real-life situations, in which an accurate response to critical, moving objects is crucial, e.g., when crossing a busy road as a pedestrian. 


\section{Acknowledgements}

This work was supported by the Swiss National Science Foundation [Grants No.320030_140696/1535 16-790; PZ00P3_154714/1 to DC]. 


\section{References}

Allen, L. M., Hasso, A. N., Handwerker, J., \& Farid, H. (2012). Sequence-specific MR imaging findings that are useful in dating ischemic stroke. Radiographics, 32(5), 1285-1297. doi: 10.1148/rg.325115760.

Azouvi, P. (1996). Functional Consequences and Awareness of Unilateral Neglect: Study of an Evaluation Scale. Neuropsychological Rehabilitation, 6(2), 133-150. doi:10.1080/713755501

Azouvi, P., Bartolomeo, P., Beis, J.-M., Perrenou, D., Pradat-Diehl, P., \& Rousseaux (2006). A battery of tests for the quantitative assessment of unilateral neglect. Restorative Neurology and Neuroscience, 24, 273-285.

Azouvi, P., Olivier, S., de Montety, G., Samuel, C., Louis-Dreyfus, A., \& Tesio, L. (2003). Behavioral assessment of unilateral neglect: study of the psychometric properties of the Catherine Bergego Scale. Archives of Physical Medicine and Rehabilitation, 84(1), 51-57. doi:10.1053/apmr.2003.50062

Behrmann, M., Watt, S., Black, S. E., \& Barton, J. J. (1997). Impaired visual search in patients with unilateral neglect: an oculographic analysis. Neuropsychologia, 35(11), $1445-1458$.

Benson, V., Ietswaart, M., \& Milner, D. (2012). Eye movements and verbal report in a single case of visual neglect. PLoS One, 7(8): e43743. doi:10.1371/journal.pone.0043743

Bourgeois, A., Chica, A. B., Migliaccio, R., Bayle, D. J., Duret, C., Pradat-Diehl, P., . . Bartolomeo, P. (2015). Inappropriate rightward saccades after right hemisphere damage: Oculomotor analysis and anatomical correlates. Neuropsychologia, 73: 1-11.

Butter, C. M., Kirsch, N. L., \& Reeves, G. (1990). The effect of lateralized dynamic stimuli on unilateral spatial neglect following right-hemisphere lesions. Restorative Neurology and Neuroscience, 2(1), 39-46. 
Cazzoli, D., Nyffeler, T., Hess, C. W., \& Müri, R. M. (2011). Vertical bias in neglect: a question of time? Neuropsychologia, 49(9), 2369-2374.

Chedru, F., Leblanc, M., \& Lhermitte, F. (1973). Visual searching in normal and braindamaged subjects (contribution to the study of unilateral inattention). Cortex, 9(1), 94111.

Ferber, S., \& Karnath, H. O. (2001). How to assess spatial neglect - Line bisection or cancellation tasks? Journal of Clinical and Experimental Neuropsychology, 23(5), 599-607. doi:10.1076/jcen.23.5.599.1243

Gainotti, G., De Luca, L., Figliozzi, F., \& Doricchi, F. (2009). The influence of distracters, stimulus duration and hemianopia on first saccade in patients with unilateral neglect. Cortex, 45(4), 506-516.

Gauthier, L., Dehaut, F., \& Joanette, Y. (1989). The Bells test: A quantitative and qualitative test for visual neglect. International Journal of Clinical Neuropsychology, 11, 49-54.

Hardiess, G., Papageorgiou, E., Schiefer, U., \& Mallot, H. A. (2010). Functional compensation of visual field deficits in hemianopic patients under the influence of different task demands. Vision Research, 50(12), 1158-1172. doi:10.1016/j.visres.2010.04.004

Heilman, K. M., Watson, R. T., \& Valenstein, E. (2003). Neglect and related disorders. In K. M. Heilman \& E. Valenstein (Eds.), Clinical neuropsychology (4 ed., pp. 296-346). London, UK: Oxford University Press.

Hopfner, S., Kesselring, S., Cazzoli, D., Gutbrod, K., Laube-Rosenpflanzer, A., Chechlacz, M., ... Nyffeler, T. (2015). Neglect and Motion Stimuli - Insights from a Touchscreen-Based Cancellation Task. PLoS One, 10(7), e0132025. doi:10.1371/journal.pone.0132025

Hunt, A. R., \& Kingstone, A. (2003). Covert and overt voluntary attention: linked or independent? Cognitive Brain Research, 18(1), 102-105. 
Ishiai, S., Furukawa, T., \& Tsukagoshi, H. (1987). Eye-fixation patterns in homonymous hemianopia and unilateral spatial neglect. Neuropsychologia, 25(4), 675-679.

Jäger, M., Nyffeler, T., Müri, R. M., Mosimann, U. P., \& Nef, T. (2015). Adapting a Driving Simulator to Study Pedestrians' Street-Crossing Decisions: A Feasibility Study. Assistive Technology, 27(1), 1-8. doi:10.1080/10400435.2014.929193

Karnath, H. O. (1988). Deficits of attention in acute and recovered visual hemi-neglect. Neuropsychologia, 26(1), 27-43.

Karnath, H. O. (1994). Spatial limitation of eye movements during ocular exploration of simple line drawings in neglect syndrome. Cortex, 30(2), 319-330.

Karnath, H. O., \& Fetter, M. (1995). Ocular space exploration in the dark and its relation to subjective and objective body orientation in neglect patients with parietal lesions. Neuropsychologia, 33(3), 371-377.

Karnath, H. O., Fetter, M., \& Dichgans, J. (1996). Ocular exploration of space as a function of neck proprioceptive and vestibular input - observations in normal subjects and patients with spatial neglect after parietal lesions. Experimental Brain Research, 109(2), 333342.

Karnath, H. O., Fruhmann Berger, M., Küker, W., \& Rorden, C. (2004). The anatomy of spatial neglect based on voxelwise statistical analysis: a study of 140 patients. Cerebral Cortex, 14(10), 1164-1172.

Karnath H. O., Himmelbach, M., \& Rorden, C. (2002). The subcortical anatomy of human spatial neglect: putamen, caudate nucleus and pulvinar. Brain, 125(Pt 2), 350-360.

Karnath, H. O., \& Niemeier, M. (2002). Task-dependent differences in the exploratory behaviour of patients with spatial neglect. Neuropsychologia, 40(9), 1577-1585.

Karnath, H. O., Niemeier, M., \& Dichgans, J. (1998). Space exploration in neglect. Brain, 121 ( Pt 12), 2357-2367. 
Kerkhoff, G., Bucher, L., Brasse, M., Leonhart, E., Holzgraefe, M., Volzke, V., . . Reinhart, S. (2014). Smooth Pursuit "Bedside" Training Reduces Disability and Unawareness During the Activities of Daily Living in Neglect: A Randomized Controlled Trial. Neurorehabilitation and Neural Repair. doi:10.1177/1545968313517757

Kerkhoff, G., \& Schindler, I. (1997). Hemi-neglect versus hemianopia. Differential diagnosis. Fortschritte der Neurologie-Psychiatrie, 65(6), 278-289. doi:10.1055/s-2007-996332

Làdavas, E., Zeloni, G., Zaccara, G., \& Gangemi, P. (1997). Eye movements and orienting of attention in patients with visual neglect. Journal of Cognitive Neuroscience, 9(1): 6774.

Land, M. F. (2006). Eye movements and the control of actions in everyday life. Progress in Retinal and Eye Research, 25(3), 296-324. doi:10.1016/j.preteyeres.2006.01.002 Mattingley, J. B., Bradshaw, J. L., \& Bradshaw, J. A. (1994). Horizontal visual-motion modulates focal attention in left unilateral spatial neglect. Journal of Neurology Neurosurgery and Psychiatry, 57(10), 1228-1235. doi:10.1136/jnnp.57.10.1228

Mattingley, J. B., Bradshaw, J. L., Bradshaw, J. A., \& Nettleton, N. C. (1994). Residual rightward attentional bias after apparent recovery from right hemisphere damage: implications for a multicomponent model of neglect. Journal of Neurology Neurosurgery and Psychiatry, 57(5), 597-604.

Mort, D. J., \& Kennard, C. (2003). Visual search and its disorders. Current Opinion in Neurology, 16(1), 51-57. doi:10.1097/01.wco.0000053590.70044.c5

Müller-Oehring, E. M., Kasten, E., Poggel, D. A., Schulte, T., Strasburger, H., \& Sabel, B. A. (2003). Neglect and hemianopia superimposed. Journal of Clinical and Experimental Neuropsychology, 25(8). doi:10.1076/jcen.25.8.1154.16727

Müri, R. M., Cazzoli, D., Nyffeler, T., \& Pflugshaupt, T. (2009). Visual exploration pattern in hemineglect. Psychological Research, 73(2), 147-157. doi:10.1007/s00426-008-02040 
Niemeier, M., \& Karnath, H. O. (2000). Exploratory saccades show no direction-specific deficit in neglect. Neurology, 54(2), 515-518.

Nyffeler, T., Hubl, D., Wurtz, P., Wiest, R., Hess, C. W., \& Müri, R. M. (2011). Spontaneous recovery of visually-triggered saccades after focal lesions of the frontal and parietal eye fields: a combined longitudinal oculomotor and fMRI study. Clinical Neurophysiology, 122(6), 1203-1210. doi:10.1016/j.clinph.2010.08.026

Nyffeler, T., Müri, R. M., Pflugshaupt, T., Wartburg, R., \& Hess, C. W. (2006). Cortical reorganization after brain damage: the oculomotor model. European Journal of Neuroscience, 23(5), 1397-1402. doi:10.1111/j.1460-9568.2006.04648.x

Olk, B., Harvey, M., \& Gilchrist, I. D. (2002). First saccades reveal biases in recovered neglect. Neurocase, 8(4), 306-313.

Pambakian, A. L., Wooding, D. S., Patel, N., Morland, A. B., Kennard, C., \& Mannan, S. K. (2000). Scanning the visual world: a study of patients with homonymous hemianopia. Journal of Neurology Neurosurgery and Psychiatry, 69(6), 751-759.

Papageorgiou, E., Hardiess, G., Ackermann, H., Wiethoelter, H., Dietz, K., Mallot, H. A., \& Schiefer, U. (2012). Collision avoidance in persons with homonymous visual field defects under virtual reality conditions. Vision Research, 52(1), 20-30. doi:10.1016/j.visres.2011.10.019

Pflugshaupt, T., Bopp, S. A., Heinemann, D., Mosimann, U. P., von Wartburg, R., Nyffeler, T., ... Müri, R. M. (2004). Residual oculomotor and exploratory deficits in patients with recovered hemineglect. Neuropsychologia, 42(9), 1203-1211. doi:10.1016/j.neuropsychologia.2004.02.002

Pizzamiglio, L., Antonucci, G., Judica, A., Montenero, P., Razzano, C., \& Zoccolotti, P. (1992). Cognitive rehabilitation of the hemineglect disorder in chronic patients with unilateral right brain damage. Journal of Clinical and Experimental Neuropsychology, 14(6), 901-923. doi:10.1080/01688639208402543 
Plummer, P., Dunai, J., \& Morris, M. E. (2006). Understanding the effects of moving visual stimuli on unilateral neglect following stroke. Brain and Cognition, 60(2), 156-165. doi: http://dx.doi.org/10.1016/j.bandc.2005.11.001

Ro, T., Rorden, C., Driver, J., \& Rafal, R. (2001). Ipsilesional biases in saccades but not perception after lesions of the human inferior parietal lobule. Journal of Cognitive Neuroscience, 13(7), 920-929.

Rojkova, K., Volle, E., Urbanski, M., Humbert, F., Dell'Acqua, F., \& Thiebaut de Schotten, M. (2016). Atlasing the frontal lobe connections and their variability due to age and education: a spherical deconvolution tractography study. Brain Structure and Function, 221(3), 1751-1766.

Rorden, C., \& Brett, M. (2000). Stereotaxic display of brain lesions. Behavioural Neurology, 12(4), 191-200.

Rorden, C., Karnath, H. O., \& Bonilha, L. (2007). Improving lesion-symptom mapping. Journal of Cognitive Neuroscience, 19(7), 1081-1088.

Sprenger, A., Kompf, D., \& Heide, W. (2002). Visual search in patients with left visual hemineglect. Progress in Brain Research, 140, 395-416. doi:10.1016/s00796123(02)40065-9

Stone, S. P., Patel, P., Greenwood, R. J., \& Halligan, P. W. (1992). Measuring visual neglect in acute stroke and predicting its recovery: the visual neglect recovery index. Journal of Neurology, Neurosurgery and Psychiatry, 55(6), 431-436.

Tant, M. L., Cornelissen, F. W., Kooijman, A. C., \& Brouwer, W. H. (2002). Hemianopic visual field defects elicit hemianopic scanning. Vision Research, 42(10), 1339-1348.

Thiebaut de Schotten, M., Ffytche, D. H., Bizzi, A., Dell'Acqua, F., Allin, M., Walshe, M., . . . Catani, M. (2011). Atlasing location, asymmetry and inter-subject variability of white matter tracts in the human brain with MR diffusion tractography. Neuroimage, 54(1), 49-59. doi:10.1016/j.neuroimage.2010.07.055 
Thiebaut de Schotten, M., Tomaiuolo, F., Aiello, M., Merola, S., Silvetti, M., Lecce, F., . . . Doricchi, F. (2014). Damage to white matter pathways in subacute and chronic spatial neglect: a group study and 2 single-case studies with complete virtual "in vivo" tractography dissection. Cerebral Cortex, 24(3), 691-706. doi:10.1093/cercor/bhs351

Werhahn, K. J., Conforto, A. B., Kadom, N., Hallett, M., \& Cohen, L. G. (2003).

Contribution of the ipsilateral motor cortex to recovery after chronic stroke. Annals in Neurology, 54(4), 464-472. doi:10.1002/ana.10686

Wilson, B., Cockburn, J., \& Halligan, P. W. (1987). The Behavioural Inattention Test. Bury St. Edmunds, UK: Thames Valley Test Company.

Zihl, J. (1995). Visual scanning behavior in patients with homonymous hemianopia. Neuropsychologia, 33(3), 287-303. 


\section{Figure captions}

\section{Fig.1. Lesion overlap maps in the three group of patients}

Lesion overlap maps in the group of patients with neglect and a probable visual field defect (N+ VFD+, upper row), in the group of patients with a visual field defect but no neglect (VFD+ N-, middle row), and in the group of patients with a right-hemispheric lesion but without any neglect or VFD (N- VFD-, lower row). Lesion overlap maps are plotted onto axial slices of the ch2 template of the Montreal Neurological Institute (MNI) brain, oriented according to the neurological convention. The slices are depicted in ascending steps of $5 \mathrm{~mm}$ (the numbers at the bottom of the figure indicate the z-position of each axial slice in the MNI Talairach stereotaxic space). The number of patients with damage to a specific brain region is colour-coded, as illustrated by the legend at the top right of the figure. The depiction of the right optic radiation (in blue) is based on a recently published DTI atlas (Thiebaut de Schotten et al., 2011; probability for voxels to belong to the right optic radiation set at > 50\%).

\section{Fig.2. Example of the virtual traffic scene in the dynamic condition}

The image of the virtual traffic scene projected onto the three canvases in the dynamic condition, with cars moving from the left and the right on the main road.

\section{Fig.3. Schematic representation of the experimental setup}

Schematic top-down view of the experimental setup with the three screens (1-3), and the three projectors $\left(\mathrm{P}_{1-3}\right)$. Adapted from: Jäger, M., Nyffeler, T., Müri, R. M., Mosimann, U. P., \& Nef, T. (2015). Adapting a Driving Simulator to Study Pedestrians' Street-Crossing Decisions: A Feasibility Study. Assistive Technology, 27(1), 1-8.

Fig. 4. Mean gaze position during early attentional orienting in the static and dynamic conditions

Mean gaze position during early attentional orienting (i.e., during the first second of visual exploration) in the four groups of participants. N+ VFD+: patients with neglect and a 
probable visual field defect (VFD) due to an additional lesion of the optic radiation; VFD+ N: patients with a VFD, but no neglect; N- VFD-: patients with a right-hemispheric lesion without any neglect or VFD. The visual scene subtended 1500 pixels on the horizontal axis. The grey vertical line (at 750 pixels) represents the middle of the visual scene on the horizontal dimension. Error bars represent the standard error of the mean (SEM). Asterisks depict significant post-hoc tests $(* p<.05)$.

\section{Fig. 5. Percentage cumulative fixation duration (CFD) in the static and dynamic conditions}

Mean percentage CFD in the static (A) and the dynamic (B) conditions, over the six columns (exploration space divided in: leftmost, left, left central, right central, right, rightmost), in the four groups of participants. N+VFD+: patients with neglect and a probable visual field defect (VFD) due to an additional lesion of the optic radiation; VFD+ $\mathrm{N}$-: patients with a VFD, but no neglect; N- VFD-: patients with a right-hemispheric lesion without any neglect or VFD. Error bars represent the standard error of the mean (SEM). Asterisks depict significant posthoc tests $(* p<.05 ; * * p<.001)$. 
Table 1: Demographic and clinical details of the patients

\begin{tabular}{|c|c|c|c|c|c|c|c|c|c|c|c|}
\hline \multirow{2}{*}{$\begin{array}{l}\text { Pat } \\
\text { Nb. }\end{array}$} & \multirow[b]{2}{*}{ Age/Sex } & \multirow[b]{2}{*}{ Etiology } & \multirow[b]{2}{*}{ Group } & \multirow{2}{*}{$\begin{array}{c}\text { Time since } \\
\text { lesion } \\
\text { (days) }\end{array}$} & \multirow{2}{*}{$\begin{array}{l}\text { Nb. voxels } \\
\text { (vol., cc.) } \\
\text { lesion } \\
\text { overlap } \\
\text { with right } \\
\text { opt. rad. }\end{array}$} & \multicolumn{4}{|c|}{ Cancellation Test } & \multirow{2}{*}{$\begin{array}{c}\begin{array}{c}\text { Line } \\
\text { Bisection }\end{array} \\
\begin{array}{c}\text { Mean } \\
\text { deviation } \\
\text { L/R }(\%)\end{array}\end{array}$} & \multirow{2}{*}{$\begin{array}{c}\begin{array}{c}\text { Catherine } \\
\text { Bergego Scale }\end{array} \\
\begin{array}{c}\text { Total score } \\
(\text { range }, 0-30)\end{array}\end{array}$} \\
\hline & & & & & & Type $^{\text {b }}$ & $\begin{array}{c}\text { No. left } \\
\text { omiss. / no. } \\
\text { left targ. }\end{array}$ & $\begin{array}{l}\text { No. right } \\
\text { omiss. / no. } \\
\text { right targ. }\end{array}$ & $\begin{array}{c}\text { Omissions } \\
\text { left minus } \\
\text { right }^{\mathrm{e}}\end{array}$ & & \\
\hline 1 & $44 / \mathrm{m}$ & Isch & Neglect & 97 & $292(.29)$ & Bells & $0 / 17$ & $0 / 17$ & 0 & 24.28 & 13 \\
\hline 2 & $55 / f$ & Hem & Neglect & 1382 & $285(.29)$ & Bells & $0 / 17$ & $0 / 17$ & 0 & -1.26 & 6 \\
\hline 3 & $59 / \mathrm{m}$ & Isch & Neglect & 1551 & $1572(1.57)$ & Bells & $1 / 17$ & $1 / 17$ & 0 & 8.35 & 3 \\
\hline 4 & $69 / \mathrm{m}$ & Hem/Isch & Neglect & 41 & $282(.28)$ & Bells & $6 / 17$ & $2 / 17$ & 4 & 10.46 & 12 \\
\hline 5 & $64 / \mathrm{m}$ & Isch & Neglect & 1137 & $4191(4.19)$ & Bells & $1 / 17$ & $0 / 17$ & 1 & -2.45 & 6 \\
\hline 6 & $49 / f$ & Hem & Neglect & 75 & $3647(3.65)$ & Bells & $3 / 17$ & $0 / 17$ & 3 & 9.76 & 15 \\
\hline 7 & $51 / \mathrm{m}$ & Isch & Neglect & 30 & $6141(6.14)$ & Bells & $3 / 17$ & $0 / 17$ & 3 & 13.25 & 13 \\
\hline 8 & $44 / \mathrm{m}$ & Hem & Neglect & 51 & $2050(2.05)$ & Bells & $0 / 17$ & $0 / 17$ & 0 & 1.54 & 9 \\
\hline 9 & $60 / f$ & Isch & L sup. quadrantanopia & 46 & $2300(2.30)$ & Bells & $0 / 17$ & $0 / 17$ & 0 & -0.31 & 0 \\
\hline 10 & $36 / f$ & Hem/Isch & L inf. quadrantanopia & 52 & $165(0.17)$ & Bells & $1 / 17$ & $0 / 17$ & 1 & 0.69 & 0 \\
\hline 11 & $60 / f$ & Hem & L inf. quadrantanopia & 1114 & $2072(2.07)$ & Bells & $0 / 17$ & $0 / 17$ & 0 & -4.27 & 0 \\
\hline 12 & $66 / \mathrm{m}$ & Hem & L hemianopia & 616 & $165(0.17)$ & Bells & $1 / 17$ & $0 / 17$ & 1 & -17.7 & 0 \\
\hline 13 & $55 / \mathrm{m}$ & Isch & L inf. quadrantanopia & 1221 & $3659(3.66)$ & Bells & $0 / 17$ & $0 / 17$ & 0 & 4.42 & 0 \\
\hline 14 & $30 / f$ & Isch & L inf. quadrantanopia & 959 & $643(0.64)$ & Bells & $0 / 17$ & $0 / 17$ & 0 & -4.96 & 0 \\
\hline 15 & $70 / \mathrm{m}$ & Isch & L hemianopia & 982 & 9585 (9.59) & Bells & $1 / 17$ & $1 / 17$ & 0 & -2.05 & 0 \\
\hline 16 & $76 / \mathrm{m}$ & Isch & L inf. quadrantanopia & 1599 & $2977(2.98)$ & Bells & $0 / 17$ & $0 / 17$ & 0 & 3.03 & 0 \\
\hline 17 & $67 / f$ & Isch & R.-hemi w/out & 49 & $0(0)$ & Bells & $1 / 17$ & $1 / 17$ & 0 & $\mathrm{n} / \mathrm{a}$ & 0 \\
\hline 18 & $63 / \mathrm{m}$ & Isch & R.-hemi w/out & 33 & $0(0)$ & Bells & $1 / 17$ & $2 / 17$ & -1 & 0.54 & 0 \\
\hline 19 & $55 / \mathrm{m}$ & Hem & R.-hemi w/out & 1737 & $0(0)$ & Bells & $1 / 17$ & $0 / 17$ & 1 & 0.03 & 0 \\
\hline 20 & $25 / \mathrm{m}$ & Hem & R.-hemi w/out & 82 & $0(0)$ & Bells & $0 / 17$ & $0 / 17$ & 0 & -6.1 & 0 \\
\hline 21 & $55 / \mathrm{m}$ & Isch & R.-hemi w/out & 28 & $0(0)$ & Bells & $0 / 17$ & $1 / 17$ & -1 & -0.52 & 0 \\
\hline 22 & $52 / f$ & Isch & R.-hemi w/out & 1131 & $0(0)$ & Bells & $2 / 17$ & $0 / 17$ & 2 & 1.21 & 0 \\
\hline 23 & $65 / \mathrm{m}$ & Isch & R.-hemi w/out & 32 & $0(0)$ & Star & $0 / 27$ & $0 / 17$ & 0 & 3.02 & 0 \\
\hline 24 & $30 / f$ & Hem & R.-hemi w/out & 90 & $0(0)$ & Bells & $1 / 17$ & $0 / 17$ & 1 & -3.51 & 0 \\
\hline
\end{tabular}


Note . Hem = Hemorrhage; inf. = inferior; Isch = Ischemia; L = left; R= right; R.-hemi w/out = patients with right-hemispheric lesions without neglect or visual field defects; sup. $=$ superior; VFD = Visual field defect.

${ }^{\mathrm{a}}$ Number of voxels (and corresponding volume, in cc., in parentheses) of each individual lesion map overlapping with the right optic radiation, as based on a recently published DTI atlas (Thiebaut de Schotten et al., 2011; probability for voxels to belong to the right optic radiation set at > 50\%).

${ }^{\mathrm{b}}$ Bells Test according to: Gauthier, L., Dehaut, F., \& Joanette, Y. (1989). The bells test: A quantitative and qualitative test for visual neglect. International Journal of Clinical

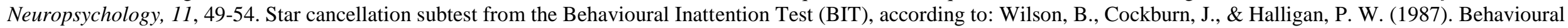
Inattention Test. Titchfield, UK: Thames Valley Test Company.

${ }^{\mathrm{c}}$ Number of omitted targets on the left side / Total number of targets on the left side. For the Start cancellation subtest from the Behavioural Inattention Test (BIT): cut-off set at $15 \%$ of omitted left-sided targets (according to: Ferber, S. \& Karnath, H. O. (2001). How to assess spatial neglect - line bisection or cancellation tasks? Journal of Clinical and Experimental Neuropsychology, 23(5), 599-607), i.e., 4 left-sided omissions.

${ }^{\mathrm{d}}$ Number of omitted targets on the right side / Total number of targets on the right side.

${ }^{\mathrm{e}}$ Number of omitted targets on the left side minus Number of omitted targets on the right side. For the Bells Test: cut-off set at > 2 (according to: Azouvi, P., Bartolomeo, P., Beis, J.-M., Perrenou, D., Pradat-Diehl, P., \& Rousseaux, M. (2006). A battery of tests for the quantitative assessment of unilateral neglect. Restorative Neurology and Neuroscience, 24, 273-285.

${ }^{\mathrm{f}}$ Mean \% deviation to right $(\mathrm{R},+)$ or left $(\mathrm{L},-)$ from the actual midline in the line bisection test. Cut-off deviation set at $11 \%$ (according to: Schenkenberg, $\mathrm{T} .$, Bradford, D. C., Ajax, E. T. (1980). Line bisection and unilateral visual neglect in patients with neurologic impairment. Neurology, 30, 509-517).

${ }^{\mathrm{g}}$ A total score on the Catherine Bergego Scale was calculated (score range, 0-30). Mild neglect: score range, 1-10; moderate neglect: score range, 11-20; severe neglect: score range, 21-30 (according to: Azouvi, P., Olivier, S., de Montety, G., Samuel, C., Louis-Dreyfus, A., \& Tesio, L. (2003). Behavioral assessment of unilateral neglect: study of the psychometric properties of the Catherine Bergego Scale. Archives of Physical Medicine and Rehabilitation, 84(1), 51-57). 


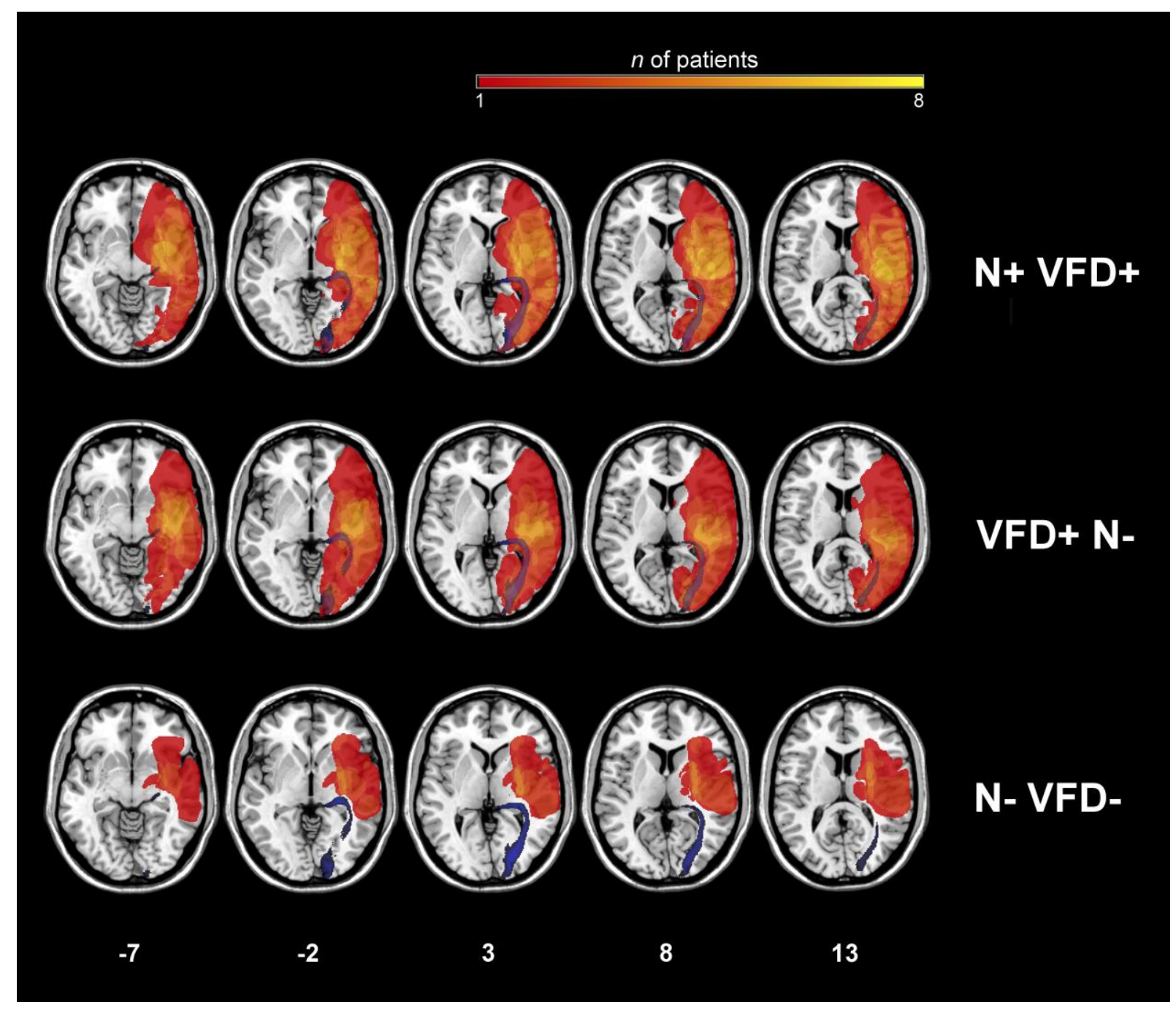




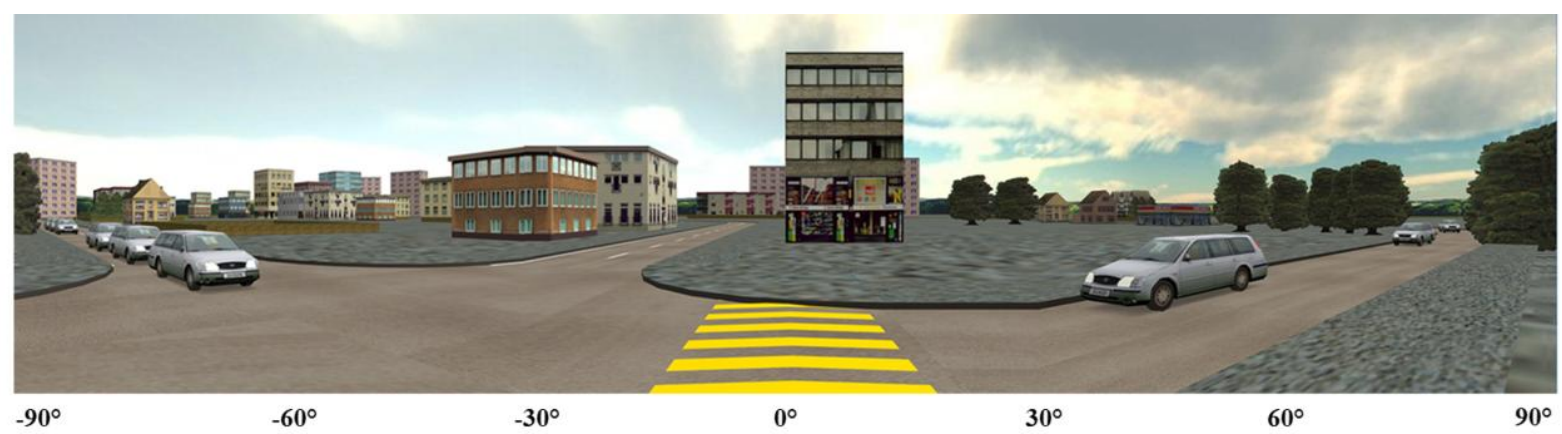




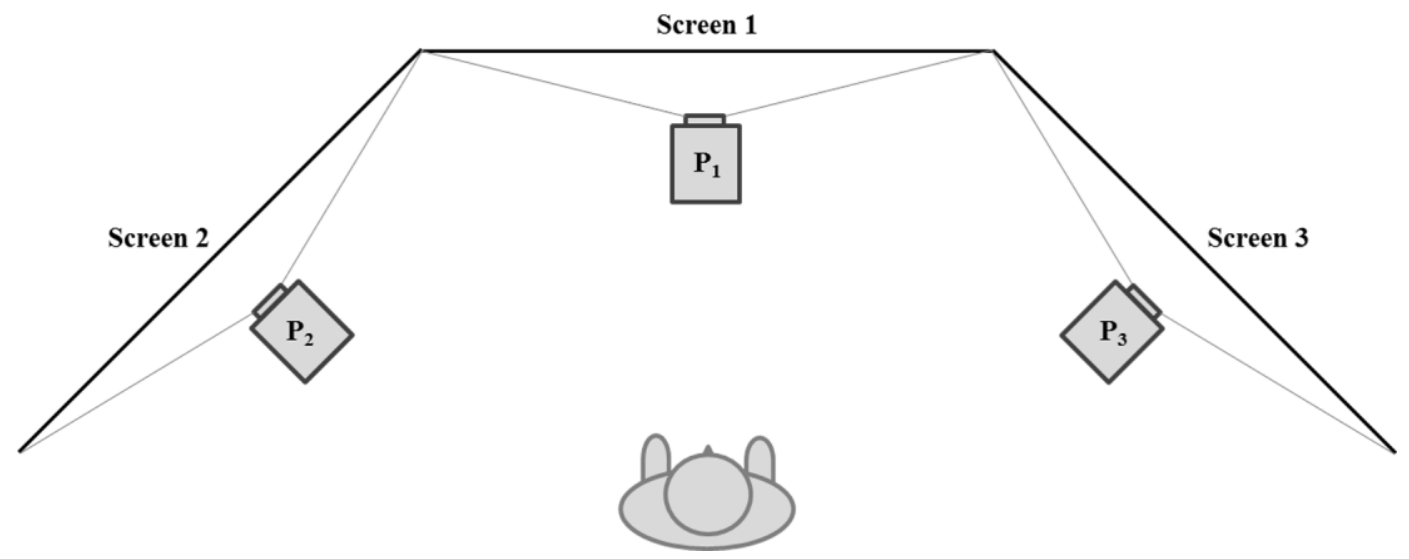

38 


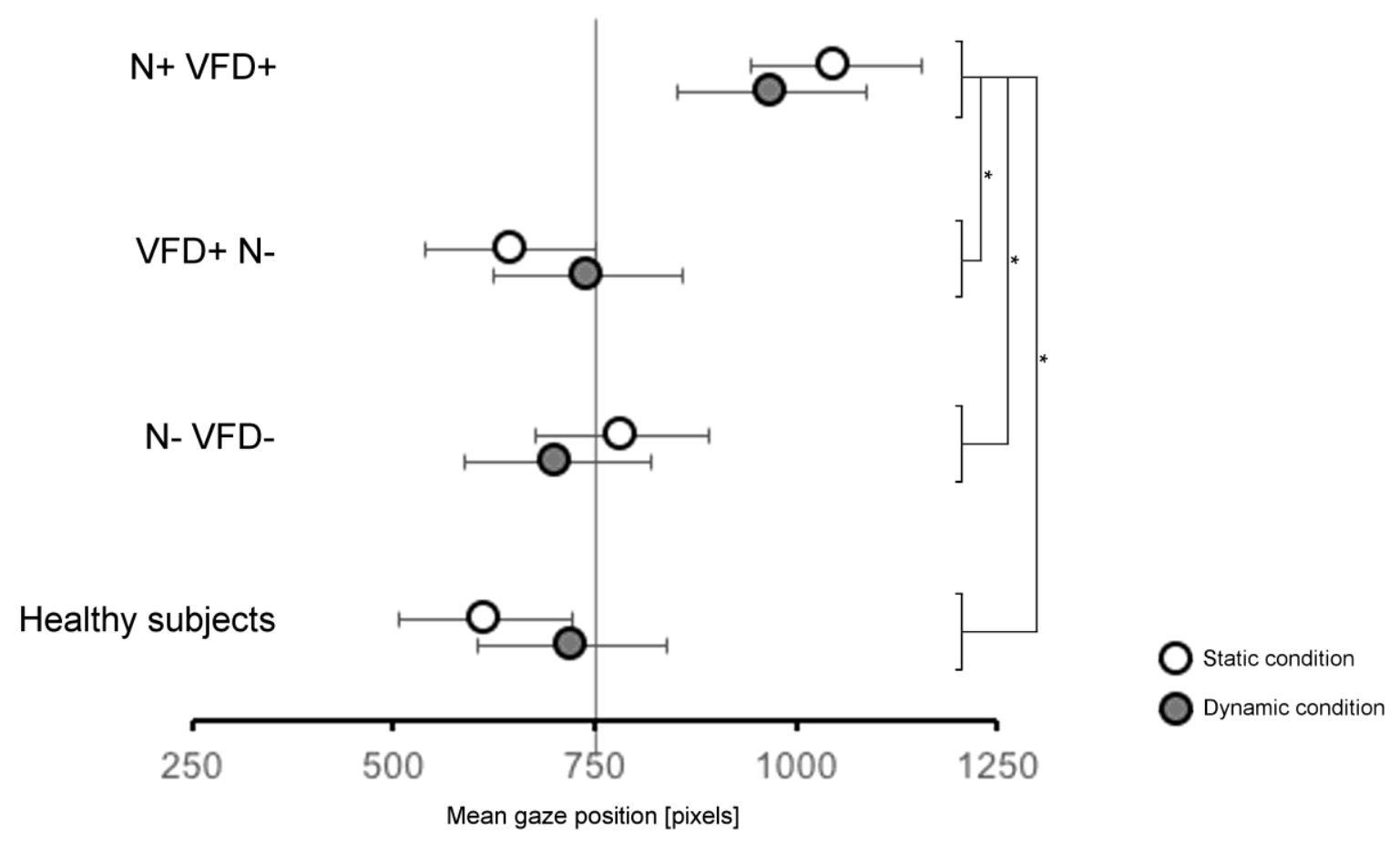


A

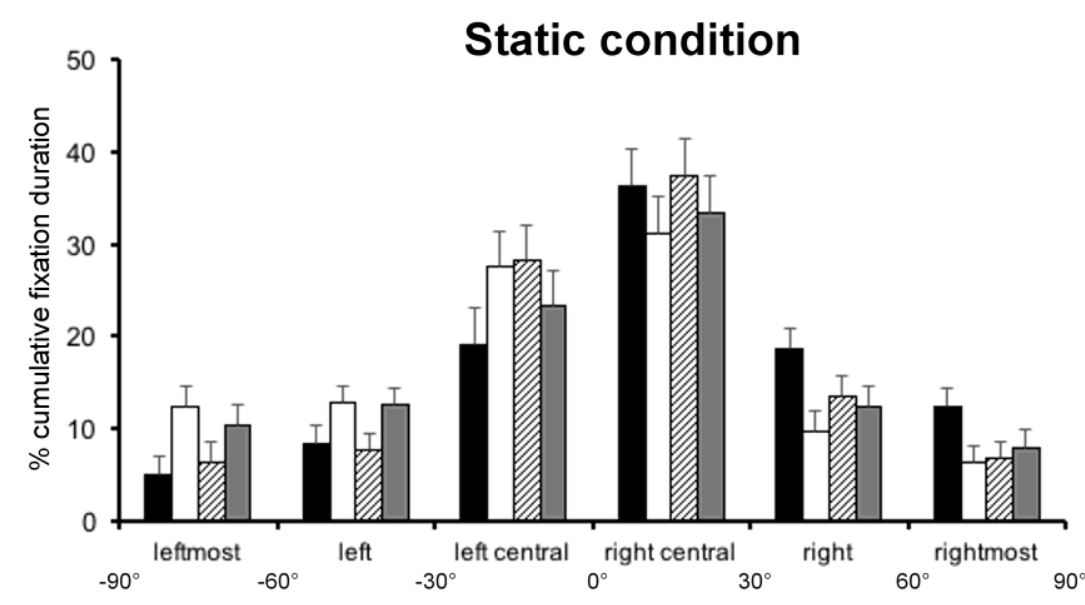

B

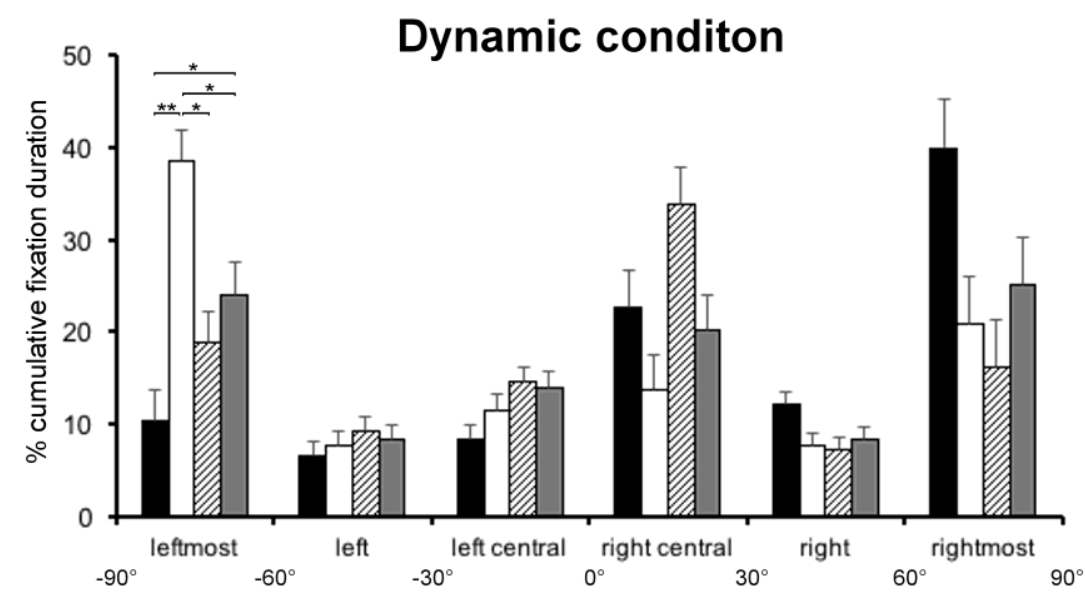

